\title{
Fundamental Study on Neutron Spectrum Unfolding using Maximum Entropy and Maximum Likelihood Method
}

\author{
Shigetaka MAEDA ${ }^{1,2^{*}}$, Hideki TOMITA ${ }^{1}$, Jun KAWARABAYASHI ${ }^{1}$ and Tetsuo IGUCHI ${ }^{1}$ \\ ${ }^{1}$ Nagoya University, Furo-cho, Chikusa-ku, Nagoya 464-8603, Japan \\ 2 Japan Atomic Energy Agency, Oarai R\&D Center, 4002, Narita O-arai Ibaraki, Japan
}

\begin{abstract}
We present a novel spectrum unfolding code, Maximum Entropy and Maximum Likelihood Unfolding Code (MEALU), based on the maximum likelihood combined with the maximum entropy method, which can determine a neutron spectrum without requiring an initial guess spectrum. We present the basic theory, limitations and assumptions built into the implementation. The performance is checked through an analysis of mock-up data. The results are compared with those obtained by conventional methods for neutron spectrum unfolding.
\end{abstract}

\section{KEYWORDS: neutron spectrum unfolding, maximum entropy method, maximum likelihood method, unde rdetermined problem}

\section{Introduction}

The inverse problem of determining the radiation source information from measured detector readings has been investigated by many researchers ${ }^{1-5}$. However, it remains unclear which method achieves high accuracy for unfolding results, especially in underdetermined problems. In addition, most spectra unfolding codes require an a priori spectrum (i.e. guess) to start the unfolding procedure for an unknown spectrum. The accuracy of the resulting spectrum strongly depends on the subjectively chosen guess spectrum.

A new spectrum unfolding code is currently under development, based on the maximum likelihood combined with the maximum entropy method. The code is called the Maximum Entropy and Maximum Likelihood Unfolding Code (MEALU). A critical advantage of the MEALU method is that it allows evaluation of the uncertainty and a determination of the neutron spectrum without an initial guess spectrum. Maximum likelihood combined with maximum entropy has been applied to the unfolding of neutron spectra, notably in the study of Itoh and Tsunoda ${ }^{1)}$. However, the MEALU formulation is different from previous applications in the way it combines maximum likelihood and maximum entropy.

It is important to estimate the error for unfold neutron spectrum as well as the integral quantity. For the sensitivity and propagation of uncertainties of the MEALU solution, we used the Monte Carlo technique to consider the measurement error and the response function uncertainty. We checked the performance of the MEALU technique by analyzing mock-up data. The results from measured data were also compared with those obtained by conventional method for neutron spectrum unfolding.

*Corresponding Author, E-mail:maeda@avocet.nucl.nagoya-u.ac.jp (C) Atomic Energy Society of Japan

\section{Inverse Problem in Radiation Measurement}

The $M$ kinds of detector response $c_{i}$ are related to the irradiated neutron spectrum $\phi(E)$ by a Fredholm integral equation of the first kind;

$$
c_{i} \approx \int_{0}^{\infty} R_{i}(E) \phi(E) d E, \quad i=1,2, \cdots, M
$$

where $R_{i}(E)$ is the response function of the $i$-th detector. The problem for neutron spectrum unfolding is to estimate $\phi(E)$ satisfying Eq.(1) with the measured data $c_{i}$ and the well evaluated data of $R_{i}(E)$ within reasonable uncertainty. The usual approach is to break the energy $E$ into discrete intervals of $N$ groups and rewrite Eq. (1) in sum (or matrix) notation as

$$
c_{i} \approx \sum_{k=1}^{N} R_{i k} \phi_{k}, \quad i=1,2, \cdots, M
$$

where $R_{i k}$ is the average value of $R_{i}(E)$ and $\phi_{k}$ is the flux in the $k$-th energy interval group. In general, since the number $N$ of energy group is larger than the number $M$ of measured detector responses, the simultaneous linear Eq. (2) results in an indefinite problem mathematically in the case of the foil activation method and multisphere neutron spectrometer (Bonner Ball).

In the underdetermined problem, the unfolding code and procedure have the following requirements:

- Stability of solution (easing the effect of measurement error)

- Uniqueness of solution (only one valid solution)

- Rejection of unphysical solutions (guarantee of a positive solution)

- Error estimation (error propagation from measurement, response function and numerical analysis).

To solve such problems, many researchers have investigated and developed numerous unfolding codes such as STAY'SL ${ }^{2)}$, FERRET ${ }^{3)}$, NEUPAC ${ }^{4)}$ and MAXED $^{5 \text { ). }}$. These codes, however, require the preparation of a 
group-wise initial guess spectrum with their covariance matrix as a priori input information, and sometimes involve very complicated calculations to obtain physically reasonable solutions when dealing with ill-conditioned matrices and/or assuming nonlinear probability functions such as lognormal distributions.

\section{The MEALU Algorithm}

\section{Definitions}

Taking the expectation of both sides of Eq. (2) and denoting the total number of neutrons incident on the detector by $\alpha$, we have

$$
a_{i}=\sum_{k=1}^{N} R_{i k} p_{k}, \quad i=1,2, \cdots, M,
$$

Where $a \equiv\langle c\rangle / \alpha$,

$$
\begin{aligned}
& p \equiv\langle\phi\rangle / \alpha, \\
& \alpha \equiv \sum\langle\phi\rangle, \\
& \sum p=1 .
\end{aligned}
$$

The expectation of a variable is denoted by putting it in brackets.

\section{Derivation of New Method}

For the vector $p$ being the probability, the quantity

$$
H=-\sum_{k=1}^{N} p_{k} \ln p_{k}
$$

is known as Shannon's information entropy. Based on the maximum entropy method, we choose the energy distribution $p$ which maximizes Eq. (8).

On the other hand, the likelihood relevant to the Poisson statistics of neutron detection ${ }^{6}$ ) is given by the following:

$$
L=\sum_{i=1}^{M}\left(c_{i} \ln <c_{i}>-\ln \left(c_{i} !\right)-<c_{i}>\right) .
$$

In this study, we select $p$ that maximizes the linear combination of the entropy and the likelihood. This new algorithm is a modification of that of Itoh and Tsunoda ${ }^{1)}$.

The combination of entropy and likelihood, $\mathrm{S}$, is given by

$$
S=H+L \text {. }
$$

The Lagrangian associated with the maximization of Eq. (10) with the constraints given by Eq. (3) and (7) is of the form

$$
\begin{aligned}
Q(\lambda) & =-\sum_{k=1}^{N} p_{k} \ln p_{k} \\
& +\sum_{i=1}^{M}\left(c_{i} \ln <c_{i}>-\ln \left(c_{i} !\right)-<c_{i}>\right), \\
& +\lambda_{0}\left(1-\sum_{k} p_{k}\right) \\
& +\sum_{i} \lambda_{i}\left(c_{i}-\sum_{k} \alpha R_{i, k} p_{k}\right)
\end{aligned}
$$

where $\lambda$ and $\lambda_{0}$ are Lagrange multipliers. Differentiating $Q$ with respect to $\left\langle c_{i}>\right.$ and $p_{k}$, and setting the result equal to zero givens the followings:

$$
\begin{aligned}
& \lambda_{i}=1-\frac{a_{i}}{\left\langle a_{i}\right\rangle}, i=1, \cdots, M, \\
& -\left(\ln p_{k}+1\right)-\lambda_{0}-(\alpha+1) \sum_{i} \lambda_{i} R_{i k}=0 .
\end{aligned}
$$

We redefine $\lambda^{\prime}$ as

$$
\lambda_{i}^{\prime}=(\alpha+1) \lambda_{i} \text {. }
$$

Finally, we obtain the set of $(N+M)$ equations to be solved in terms of $\lambda_{i}^{\prime}$ :

$$
\begin{aligned}
& p_{k}=\frac{1}{Z\left(\lambda^{\prime}\right)} \exp \left(-\sum_{i=1}^{M} \lambda_{i}^{\prime} R_{i k}\right), \\
& Z\left(\lambda^{\prime}\right)=\sum_{k=1}^{N} \exp \left(-\sum_{i=1}^{M} \lambda_{i}^{\prime} R_{i k}\right), \\
& \frac{\lambda_{i}^{\prime}}{\alpha+1}=1-\frac{a_{i}}{\left\langle a_{i}\right\rangle}, \quad i=1, \cdots, M .
\end{aligned}
$$

According to Eq. (17), the number of $\lambda^{\prime}{ }_{i}$ agrees with the number of $a_{i}$ for the underdetermined and overdetermined problems. Although this paper focuses on the underdetermined problem, the MEALU method can also be applied to the overdetarmined problem without reformulation.

The problem now is to solve the nonlinear system (17) for the vector $\langle a\rangle$ included in $\lambda$ '. For this we applied Newton's method. The unfolded spectrum under the present method is not affected by an arbitrary choice of initial guess, but a starting point of $\lambda$ ' is required in the iterative scheme. We employed all zeros as this starting value, which corresponds to a flat spectrum.

The solution $\langle\phi\rangle$ was calculated from $p$ multiplied by $\alpha$ :

$$
\langle\phi\rangle=\alpha \times p \text {. }
$$

\section{Error Estimation}

In the current problem, it is difficult to apply an error propagation estimation because Eq. (17) is transcendental. We have thus considered the measurement error and response function uncertainty using the Monte Carlo technique in a statistical sense for error propagation studies.

Numerous sample detector responses $c^{(m)}$ are generated at random from a probability density around a measured detector response $c_{i}$, where $(m)$ mean the $m$-th trial. Similarly, sample response functions $R^{(m)}$ are generated around the uncertainty of the response function.

$$
\begin{aligned}
& c_{i}{ }^{(m)}=c_{i} \pm \delta c_{i}, i=1, \cdots, M, \\
& R_{i, k}{ }^{(m)}=R_{i, k}+\delta R_{i, k}, \\
& \quad i=1, \cdots, M, k=1, \cdots, N
\end{aligned} .
$$

$c^{(m)}$ and $R^{(m)}$ are then input into the MEALU and $\phi^{(m)}$ are output by the MEALU:

$$
\phi^{(m)}=M E A L U\left[R^{(m)}, c^{(m)}\right] .
$$

Then, the variance for each neutron group can be estimated as follows: 


$$
\Sigma_{\phi}=\frac{1}{N M A X-1} \sum_{m=1}^{N M A X}\left(\phi^{(m)}-\bar{\phi}^{(m)}\right)^{2}
$$

In a similar way, the integral quantities and their variances can be formulated.

\section{Numerical Test}

We created a prototype MEALU program and checked its performance through an analysis of mock-up data.

A problem with five group neutron fluxes unfolded from three detector responses was prepared as shown in Table 1. In this test, the total neutron flux $(\alpha)$ was assumed as a given parameter.

Table 1 Parameters for Test Problem

\begin{tabular}{|c|c|c|c|c|}
\hline \multirow{2}{*}{$K$} & \multicolumn{3}{|c|}{ Response Function } & \multirow{2}{*}{$\phi_{k, \text { exact }}$} \\
\hline & $\mathrm{R}_{l, k}$ & $\mathrm{R}_{2, k}$ & $\mathrm{R}_{3, k}$ & \\
\hline 1 & 2 & 0 & 0 & 8 \\
\hline 2 & 4 & 3 & 1 & 13 \\
\hline 3 & 3 & 2 & 2 & 9 \\
\hline 4 & 3 & 2 & 4 & 5 \\
\hline 5 & 2 & 2 & 4 & 2 \\
\hline$c_{i}$ & 114 & 71 & 59 & 37 \\
\hline
\end{tabular}

\section{Results and Discussion}

\section{Unfolded Results}

Table 2 and Fig. 1 show the unfold spectrum obtained from the MEALU prototype code. The detector response using the unfold spectrum is also shown in Table 3. In this case, the MEALU unfolding result is in good agreement with the exact value in spectrum shape and quantity without an initial guess spectrum. The re-fold detector responses using an unfold spectrum also agree with the exact value.

\begin{tabular}{ccc} 
Table 2 & \multicolumn{2}{c}{ Unfold Spectrum } \\
\hline$k$ & $\phi_{\text {unfold }}$ & $\phi_{\text {exact }}$ \\
\hline 1 & 8.3 & 8 \\
2 & 13.5 & 13 \\
3 & 7.4 & 9 \\
4 & 4.5 & 5 \\
5 & 3.2 & 2 \\
\hline
\end{tabular}

\begin{tabular}{ccc}
\multicolumn{2}{c}{ Table 3} & \multicolumn{2}{c}{ Unfold Response } \\
\hline$i$ & $R_{\text {unfold }}$ & $R_{\text {exact }}$ \\
\hline 1 & 112.7 & 114 \\
2 & 70.7 & 71 \\
3 & 59.1 & 59 \\
\hline
\end{tabular}

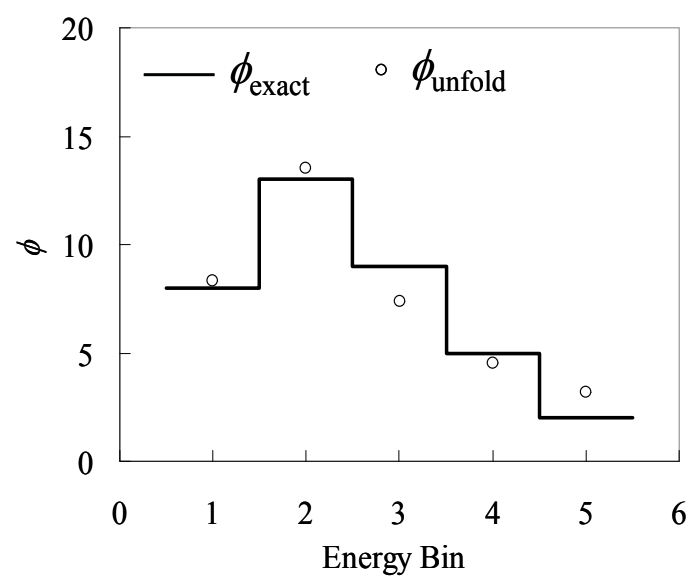

Fig. 1 Unfold Spectrum

\section{Error Estimation}

Table 4 and Fig. 2, and Table 5 and Fig. 3 show the results of error estimation by measurement error and response function uncertainty, which are obtained after 10000 trials of random walk, respectively. The Monte Carlo technique provided the probability density, shown in Fig. 4. The originals were restored within $2 \sigma$ at each energy bin.

Table 4 Result of Error Estimation by Measurement Data

\begin{tabular}{ccccc}
\hline$k$ & $\phi_{\text {unfold }}$ & $\Delta \phi_{\text {unfold }}(1 \sigma)$ & $\Delta \phi_{\text {unfold }}(\%)$ & $\phi_{\text {exact }}$ \\
\hline 1 & 8.3 & 2.4 & 28.4 & 8 \\
2 & 13.5 & 3.1 & 23.3 & 13 \\
3 & 7.4 & 1.1 & 15.4 & 9 \\
4 & 4.5 & 3.2 & 71.6 & 5 \\
5 & 3.2 & 2.9 & 90.2 & 2 \\
\hline
\end{tabular}

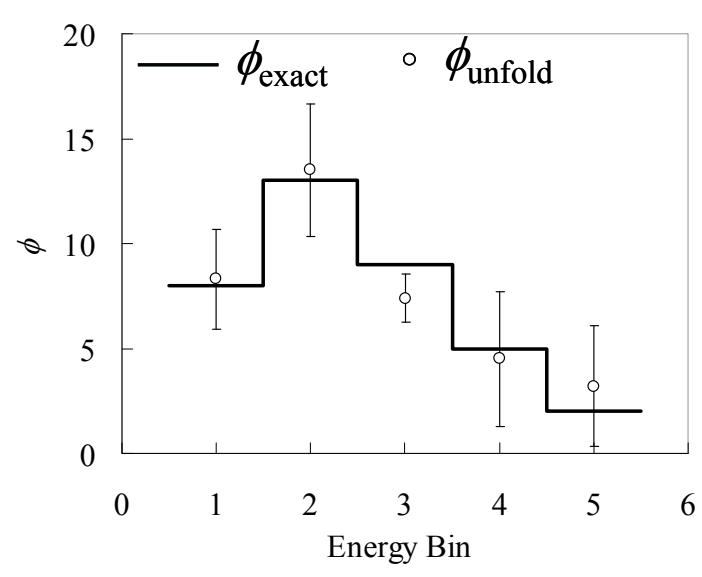

Fig. 2 Error Estimation by Measurement Error

Table 5 Result of Error Estimation by Response Function Uncertainty

\begin{tabular}{ccccc}
\hline$k$ & $\phi_{\text {unfold }}$ & $\Delta \phi_{\text {unfold }}(1 \sigma)$ & $\Delta \phi_{\text {unfold }}(\%)$ & $\phi_{\text {exact }}$ \\
\hline 1 & 8.3 & 0.12 & 1.5 & 8 \\
2 & 13.5 & 0.16 & 1.2 & 13 \\
3 & 7.4 & 0.08 & 1.1 & 9 \\
4 & 4.5 & 0.24 & 5.3 & 5 \\
5 & 3.2 & 0.23 & 7.0 & 2 \\
\hline
\end{tabular}

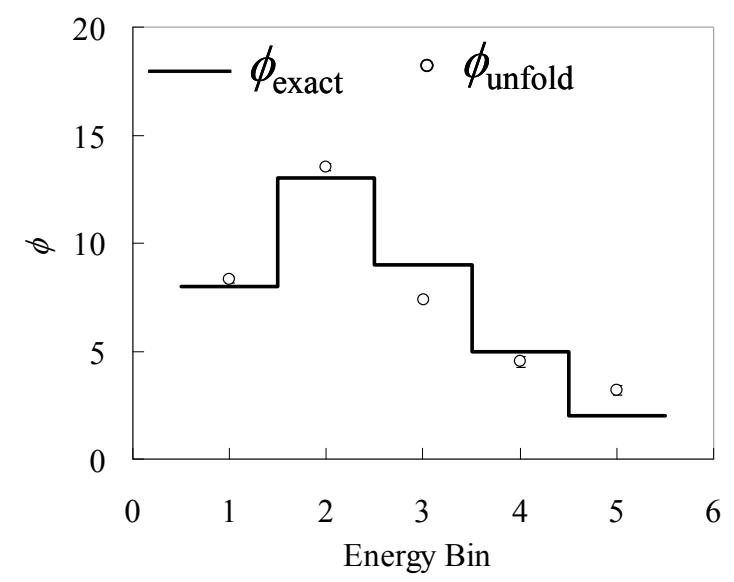

Fig. 3 Error Estimation by Response Function 

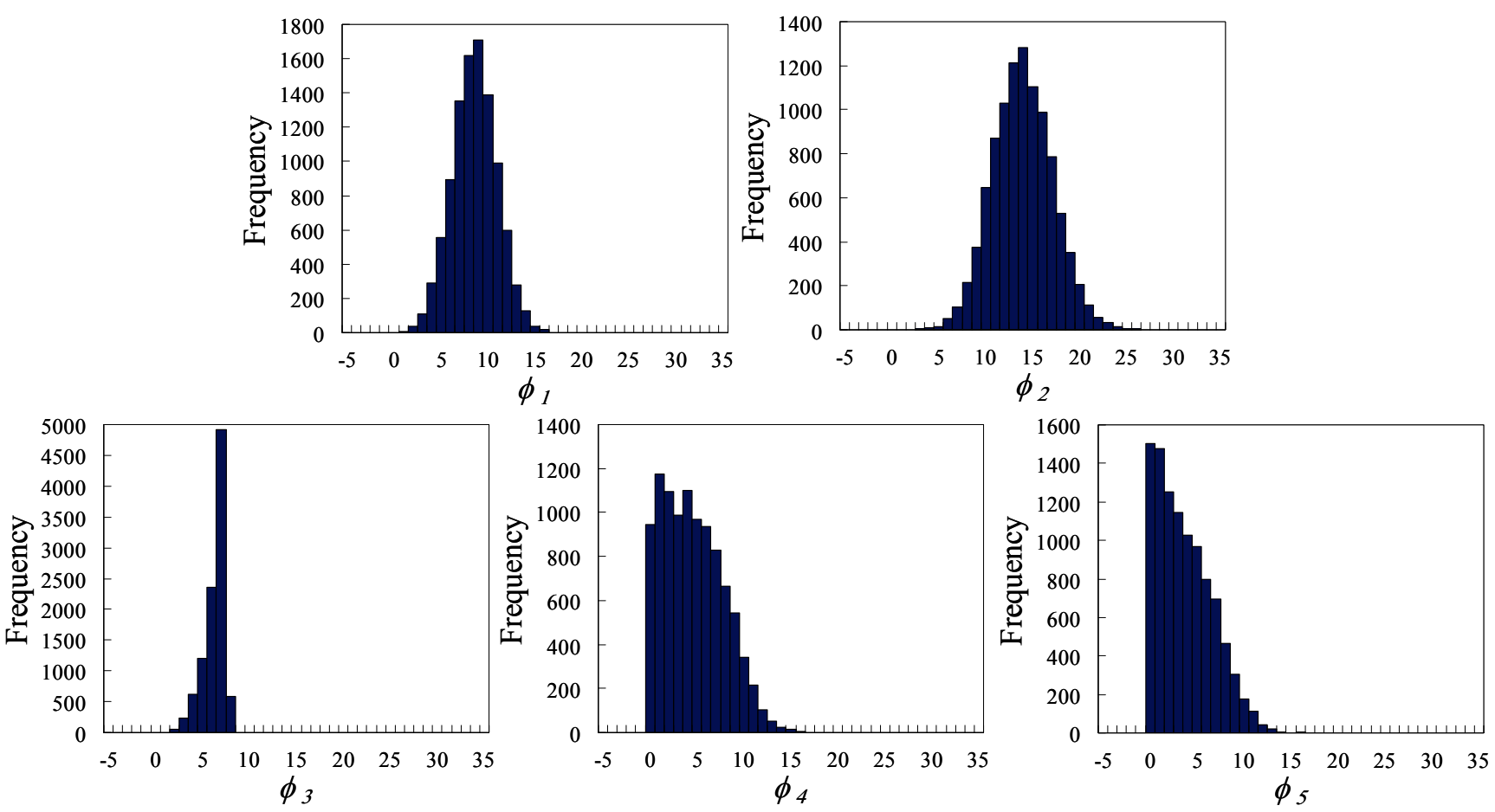

Fig. 4 A Posteriori Probability Density of Group Neutron Flux $\phi_{k}$

\section{Comparison with Conventional Method}

Table 6 shows the comparison of the unfold spectrum and its estimated error obtained by the MEALU and J-1 methods from the same detector responses, response functions and uncertainties. Both results are in good agreement quantitatively within the estimated uncertainties.

Table 6 Comparison with Conventional Method

\begin{tabular}{cccccc}
\hline \multirow{2}{*}{$k$} & \multicolumn{2}{c}{ MEALU } & \multicolumn{2}{c}{ J-1 Method } & \multirow{2}{*}{$\phi_{\text {exact }}$} \\
& $\phi_{\text {unfold }}$ & $\Delta \phi_{\text {unfold }}(1 \sigma)$ & $\phi_{\text {unfold }}$ & $\Delta \phi_{\text {unfold }}(1 \sigma)$ & \\
\hline 1 & 8.3 & 2.3 & $10.3(8)^{*}$ & 2.9 & 8 \\
2 & 13.5 & 3.1 & $10.3(13)$ & 2.2 & 13 \\
3 & 7.4 & 1.2 & $8.2(9)$ & 2.9 & 9 \\
4 & 4.5 & 3.2 & $5.2(5)$ & 2.5 & 5 \\
5 & 3.2 & 3.0 & $5.1(2)$ & 2.5 & 2 \\
\hline
\end{tabular}

* The result in case of $\Delta c(1 \sigma)=0 \%$

\section{Conclusion}

A new spectrum unfolding code, MEALU, is currently under development based on the maximum likelihood combined with the maximum entropy method. We have demonstrated typical unfolding results using mock-up data. Compared with conventional methods, the new method has the following potential advantages:

- It is not necessary to prepare an initial guess spectrum and covariance data as a priori information.

- A positive solution is guarantied.
We applied the Monte Carlo method to estimate the posterior probability distribution of the solution and obtained a reasonable error. In the future, the performance will be checked through analyses of mock-up data for several detector systems. The results from measured data will also be compared with those obtained by conventional methods for neutron spectrum unfolding.

\section{References}

1) Itoh, S. and T. Tsunoda, "Neutron Spectrum Unfolding with Maximum Entoropy and Maximum Likelihood," J. Nucl. Sci. Technol., 26[9], 833(1989).

2) F. G. Perey, ORNL/TM 6062, Oak Ridge National Laboratory, (1977).

3) F. Schmittroth, HEDL-TME 79-40, Hanford Engineering Development Laboratory, (1979).

4) Taniguchi, T., et al., "Systematic study on spectral effects in the adjustment calculations using the NEUPAC- 83 code," Proc. the Fifth Int. Symp. on Reactor Dosimetry, Geesthacht, Germany, sept. 24-28, 1984, pp. 685 (1985).

5) Reginatto, M., P. Goldhagen, et al., "Spectrum unfolding, sensitivity analysis and propagation of uncertainties with the maximum entropy deconvolution code MAXED," Nuclear Instruments and Methods in Physics Research Section A, 476(1-2), 242 (2002).

6) Itoh, S., "A Fundamental Study of Neutron Spectra Unfolding Based on The Maximum Likelihood Method," Nuclear Instruments and Methods in Physics Research A251, 144 (1986). 